\title{
Unresolved native range taxonomy complicates inferences in invasion ecology: Acacia dealbata Link as an example
}

\author{
Heidi Hirsch • Laure Gallien • Fiona A. C. Impson • Catharina Kleinjan - David M. Richardson • \\ Johannes J. Le Roux
}

Received: 17 October 2016/Accepted: 11 February 2017/Published online: 17 February 2017

(C) Springer International Publishing Switzerland 2017

\begin{abstract}
Elaborate and expensive endeavours are underway worldwide to understand and manage biological invasions. However, the success of such efforts can be jeopardised due to taxonomic uncertainty. We highlight how unresolved native range taxonomy can complicate inferences in invasion ecology using the invasive tree Acacia dealbata in South Africa as an example. Acacia dealbata is thought to comprise two subspecies based on morphological characteristics and environmental requirements within its native range in Australia: ssp. dealbata and spp. subalpina. Biological control is the most promising option for managing invasive A. dealbata populations in South Africa, but it remains unknown which genetic/taxonomic entities are
\end{abstract}

Electronic supplementary material The online version of this article (doi:10.1007/s10530-017-1381-9) contains supplementary material, which is available to authorized users.

H. Hirsch $(\bowtie) \cdot$ L. Gallien · D. M. Richardson ·

J. J. Le Roux

Centre for Invasion Biology, Department of Botany and

Zoology, Stellenbosch University,

Private Bag X1, Matieland 7602, South Africa

e-mail: heidihirsch71@googlemail.com

F. A. C. Impson

Plant Protection Research Institute,

Private Bag X5017, Stellenbosch 7599, South Africa

F. A. C. Impson - C. Kleinjan

Plant Conservation Unit, Department of Biological

Sciences, University of Cape Town, Rondebosch 7701,

South Africa present in the country. Resolving this question is crucial for selecting appropriate biological control agents and for identifying areas with the highest invasion risk. We used species distribution models (SDMs) and phylogeographic approaches to address this issue. The ability of subspecies-specific and overall species SDMs to predict occurrences in South Africa was also explored. Furthermore, as non-overlapping bioclimatic niches between the two taxonomic entities may translate into evolutionary distinctiveness, we also tested genetic distances between the entities using DNA sequencing data and network analysis. Both approaches were unable to differentiate the two putative subspecies of $A$. dealbata. However, the SDM approach revealed a potential niche shift in the non-native range, and DNA sequencing results suggested repeated introductions of different native provenances into South Africa. Our findings provide important information for ongoing biological control attempts and highlight the importance of resolving taxonomic uncertainties in invasion ecology.

Keywords DNA sequencing $\cdot$ Fabaceae $\cdot$ Species distribution models $\cdot$ Subspecies $\cdot$ Tree invasions

\section{Introduction}

Invasive species are a major threat to global biodiversity, human livelihoods, and economic development (Early et al. 2016). Elaborate and expensive 
approaches are therefore undertaken in many parts of the world to understand and manage them. Some of such endeavours, however, can only be successful and efficient when the taxonomy of the invasive species in question is resolved (Pyšek et al. 2013). Indeed, management efforts can be constrained by uncertain taxonomy, or if the taxonomic unit (e.g. species) in focus is not appropriate. Unresolved taxonomy may substantially impact on the predictive power of species distribution models (SDMs; Guisan and Zimmermann 2000), and thus the ability to predict invasion risk. For example, Ensing et al. (2013) found unreliable taxonomic identifications of Pilosella glomerata to predict a more heterogeneous niche in its non-native North American range compared to taxonomically reliable records. Similarly, in some instances invasive species should not be regarded as uniform taxonomic entities due to the presence of genetically distinct subspecies or lineages that hold different invasion histories and establishment capacities (e.g. Phragmites australis ssp. australis vs. $P$. australis ssp. americanus, Saltonstall 2002). From a management perspective, resolved taxonomy is of particular importance in biological control programs for which coevolved and hostspecific control agents need to be identified from the species' native range (Wardill et al. 2005; Pyšek et al. 2013).

Australian acacias (genus Acacia) are an ideal study system to investigate the influence of taxonomy in invasion ecology in more detail, because the intraspecific variation of several invasive acacias is characterized by pronounced geographic structure within their native range, often in the form of distinct subspecies (e.g. Ndlovu et al. 2013; Thompson et al. 2011). We focus on Acacia dealbata Link (Fabaceae), an aggressive invader of natural ecosystems in several countries including South Africa (Rejmánek and Richardson 2013), and examine how its putative native taxonomy relates to its invasive distribution in South Africa. Acacia dealbata is native to eastern and south-eastern Australia and Tasmania where it occurs on tablelands and slopes (Poynton 2009). In its native range, A. dealbata supposedly consists of two subspecies, A. dealbata ssp. dealbata and ssp. subalpina, which differ in their ecological niche requirements and to some extent in their morphology. Subspecies dealbata has been recorded at lower altitudes than ssp. subalpina: up to $1000 \mathrm{~m}$ a.s.l. versus mainly above $700 \mathrm{~m}$ a.s.l. (although down to $300 \mathrm{~m}$ a.s.l. in a few cases); with a wider range of annual precipitation: 500-1600 versus $600-700 \mathrm{~mm}$ (Kodela and Tindale 2001; www.florabank.org.au, accessed 20 February 2016). Subspecies dealbata has been described as being generally taller than ssp. subalpina (shrub- or tree-like up to $10 \mathrm{~m}$ vs. shrub-like up to $3 \mathrm{~m}$, or small tree up to $5 \mathrm{~m}$ ), having longer leaves (5-14 vs. $1.5-8.5 \mathrm{~cm})$ and longer pinnae $(1.5-5.5$ vs. $0.5-2.5[-$ 3] $\mathrm{cm}$ ) (Kodela and Tindale 2001; www.florabank. org.au). Kodela and Tindale (2001) nonetheless acknowledge the occurrence of intermediate (i.e. overlapping) stands between the two subspecies where ssp. dealbata is found as a tree at lower mountain slopes and ssp. subalpina as a shrub at upper slopes. Acacia dealbata was introduced to South Africa between 1880 and 1890, primarily for wood production (Poynton 2009). Invasive stands of the species now cover large areas of the country and have a major impact on water resources (Holmes et al. 2005). Consequently, there is an urgent need to understand its invasion success and possible future spread in more detail in order to develop more effective management and control strategies.

Although it is generally assumed that only ssp. dealbata has become naturalized and problematic outside the native range (Maslin and McDonald 2004; www.florabank.org.au), no information about the genetic structure within the native and invasive ranges, or the levels of genetic divergence between the two putative subspecies exists. It is important to know which of the sub-specific entities of $A$. dealbata is present in South Africa, particularly if each subspecies has different environmental requirements, which may influence their potential distributions. Further, as an extensive biological control programme is ongoing against this species in South Africa (Impson et al. 2011), such information is also important as it may influence the efficiency of biological control agents due to different environmental requirements or different co-evolutionary histories between host plants and potential control agents (e.g. Paterson et al. 2011).

Based on the hypothesis that the two putative $A$. dealbata subspecies should be genetically distinct and that they should occupy distinct bioclimatic niches, we employed a combination of species distribution models (SDMs) and DNA sequencing analyses to identify the actual taxonomic entities invasive in South Africa. We first investigated whether the environmental requirements of the two putative $A$. dealbata 
subspecies can be distinguished by applying SDMs. We then compared these SDMs in their ability to predict known occurrences of $A$. dealbata in its nonnative range in South Africa. Finally, using DNA sequencing data from native as well non-native South African populations, we explored levels of divergence between the putative subspecies and whether we could identify which sub-specific entity was introduced to South Africa.

\section{Materials and methods}

Data sources and preparation

Geo-referenced distribution records of A. dealbata from its native range and its non-native range in South Africa were obtained from the Atlas of Living Australia database (www.ala.org.au; accessed 14 April 2016), the Global Biodiversity Information Facility (GBIF; www.gbif.org; accessed 14 April 2016) and the Southern African Plant Invaders Atlas (SAPIA; Henderson 2007). This procedure resulted in 28,128 native Australian occurrences and 920 nonnative occurrences in South Africa. The native Australian dataset was also split to include either only ssp. subalpina (1203 records) or ssp. dealbata occurrences (9329 records). For all datasets, obvious erroneous records (i.e. non-native occurrences in Australia and those with coordinates that fell in the ocean) were manually removed and data preparation and quality assessment was performed following the recommendations of Robertson et al. (2016) in the R statistical environment ( $\mathrm{R}$ Core Team 2016). All duplicate occurrence records were removed at a spatial resolution of $\sim 5 \mathrm{~km}$ (2.5 arc minutes). After these data preparation steps, a total of 217 records (4.9\% of the total native range dataset) for ssp. subalpina, 2214 records (49.7\% of the total native range dataset) for ssp. dealbata, 4451 records for A. dealbata within the native range (2020 records of this dataset did not have a formal subspecies information), and 729 records for non-native occurrences in South Africa could be used for further analyses (Fig. 1a, d).

Bioclimatic variables

Bioclimatic variables were selected according to their ecological relevance for Australian acacia distributions, the described niche differences between the two A. dealbata subspecies, and to minimize multicollinearity (Kodela and Tindale 2001; CastroDíez et al. 2011; Thompson et al. 2011). This preselection process led to the selection of four bioclimatic variables: temperature seasonality (Bio4), maximum temperature of the warmest month (Bio5), minimum temperature of the coldest month (Bio6), and annual precipitation (Bio12). All variables were downloaded from the WorldClim database (Hijmans et al. 2005) at a resolution of $\sim 5 \mathrm{~km}$.

Species distribution models (SDMs)

For each of our three taxonomic units (ssp. subalpina, ssp. dealbata, and A. dealbata) we built a set of ensemble SDMs (Araújo and New 2007) using the R package biomod2 (Thuiller et al. 2016). First, for each dataset of occurrences in Australia, we generated three sets of random pseudo-absences, containing as many pseudo-absences as occurrence records (in order to always obtain a prevalence of 1). We then randomly split each presence/pseudo-absence datasets into two main subsets: (a) the model calibration data set including $90 \%$ of the full dataset, and (b) the ensemble model evaluation data set containing the remaining $10 \%$ of the full dataset (Marmion et al. 2009). The calibration data set was then used to model species distributions with four different algorithms: generalized linear models (GLM), generalized boosting models (GBM), generalized additive models (GAM), and random forests (RF), together with a split-sample cross-validation procedure ( $70 \%$ of the calibration set is used for "inner-calibration" of the single models, and 30\% for "inner-validation"; Marmion et al. 2009) which we repeated four times. Each single model was evaluated with four summary statistics; the true skill score statistic (TSS; Allouche et al. 2006), the area under the receiver operating characteristic plot (AUC; Swets 1988), the sensitivity (proportion of correctly predicted presences), and the specificity (proportion of correctly predicted absences). In summary, for each taxonomic unit we built 48 single models ( 3 pseudoabsences $* 4$ model algorithms $* 4$ cross-validations). Among the 48 different models generated, we selected only those with a TSS score higher than 0.8 (corresponding to models with high discriminatory power) and averaged their projections in Australia and South Africa to generate ensemble model projections 
(weighting the importance of each model by its TSS score; Araújo and New 2007). Each ensemble model was then evaluated on the "ensemble model evaluation data set" (i.e. $10 \%$ of the original dataset) with TSS, AUC, sensitivity and specificity scores. Finally, we evaluated the SDM performance at predicting the invasive populations with the sensitivity scores in South Africa.

We assumed that, if the climatic niches for the two subspecies are different, then the SDM for one subspecies would not be able to predict the occurrences of the other subspecies. In contrast, no clear climatic niche differences between the subspecies would be supported if the SDM for one subspecies can predict the occurrences of the other subspecies with high accuracy.

DNA sequencing and analyses

A total of 44 localities across the native range (Australia mainland: 29 localities; Tasmania: 15 localities) and 18 localities in the South African invaded range were sampled (one or two accessions per locality $[n=104]$; Table S1; Fig. S1, Online Resource). In the native range, specific attention was paid to sample localities of both putative subspecies which included a site (ID: AUS_23; Table S1, Online Resource) in the region where the holotype of the more restricted subspecies, ssp. subalpina (Specimen NSW376247, National Herbarium of New South Wales, Australia), was collected. Extraction of whole genomic DNA, PCR amplifications and sequencing of the external transcribed spacer region (ETS) was performed as described in the Materials and methods S1, Online Resource.

Aligned DNA sequence data were used to identify haplotypes and illustrate their relationships as a parsimony network using the $\mathrm{R}$ package pegas (Paradis 2010). This approach was chosen because of its higher resolution and suitability for investigating the relationships between intraspecific sequence samples compared to traditional phylogenetic tree approaches (Le Roux et al. 2011).

Further, haplotype richness (Hk) and absolute gene differentiation (DGst) were calculated for the two regions in the native range (Australian mainland and Tasmania) and for the non-native range in South Africa using CONTRIB (Petit et al. 1998). Because richness measures are influenced by sampling effort, we used the rarefaction approach implied in CONTRIB to account for skewness of sample sizes among our sampled regions. We applied a rarefaction to the smallest regional sample size $(n=26$, Tasmania) to achieve comparability among the regions.

\section{Results}

Species distribution models

All three ensemble models showed high performance at identifying presences and absences in the species native range (TSS $>0.9$ and AUC $>0.9$; see also Fig. S2 and Table S2, Online Resource). All ensemble models were able to predict most Australian presences of the three different taxonomic units correctly (95.1-99.5\%), except the ssp. subalpina model which predicted only $59.3 \%$ of the spp. dealbata and $65.1 \%$ of the A. dealbata occurrences correctly (Table S3, Online Resource).

All ensemble model projections in Australia displayed wide potential distributions across both regions of the native $A$. dealbata range (i.e. Australian mainland and Tasmania; Fig. 1b, c). The largest climatically suitable area was predicted by the $A$. dealbata model (Fig. 1c). Vast potential distributions were also predicted by all ensemble models when projected into South Africa, with high suitability in the eastern and south-eastern regions of the country where the current invasion hotspot of $A$. dealbata is located (Fig. 1e, f). However, none of the SDMs was able to correctly predict all known non-native occurrences of A. dealbata in South Africa: ssp. subalpina model predicted $46.5 \%$, ssp. dealbata model predicted $70.8 \%$, and the A. dealbata model predicted $61.6 \%$. Further, although the projections of the ssp. dealbata and ssp. subalpina model showed a major overlap in their predicted potential distribution in South Africa, it appears that the sub-specific models predict different suitable areas to some extent (Fig. 1e). 


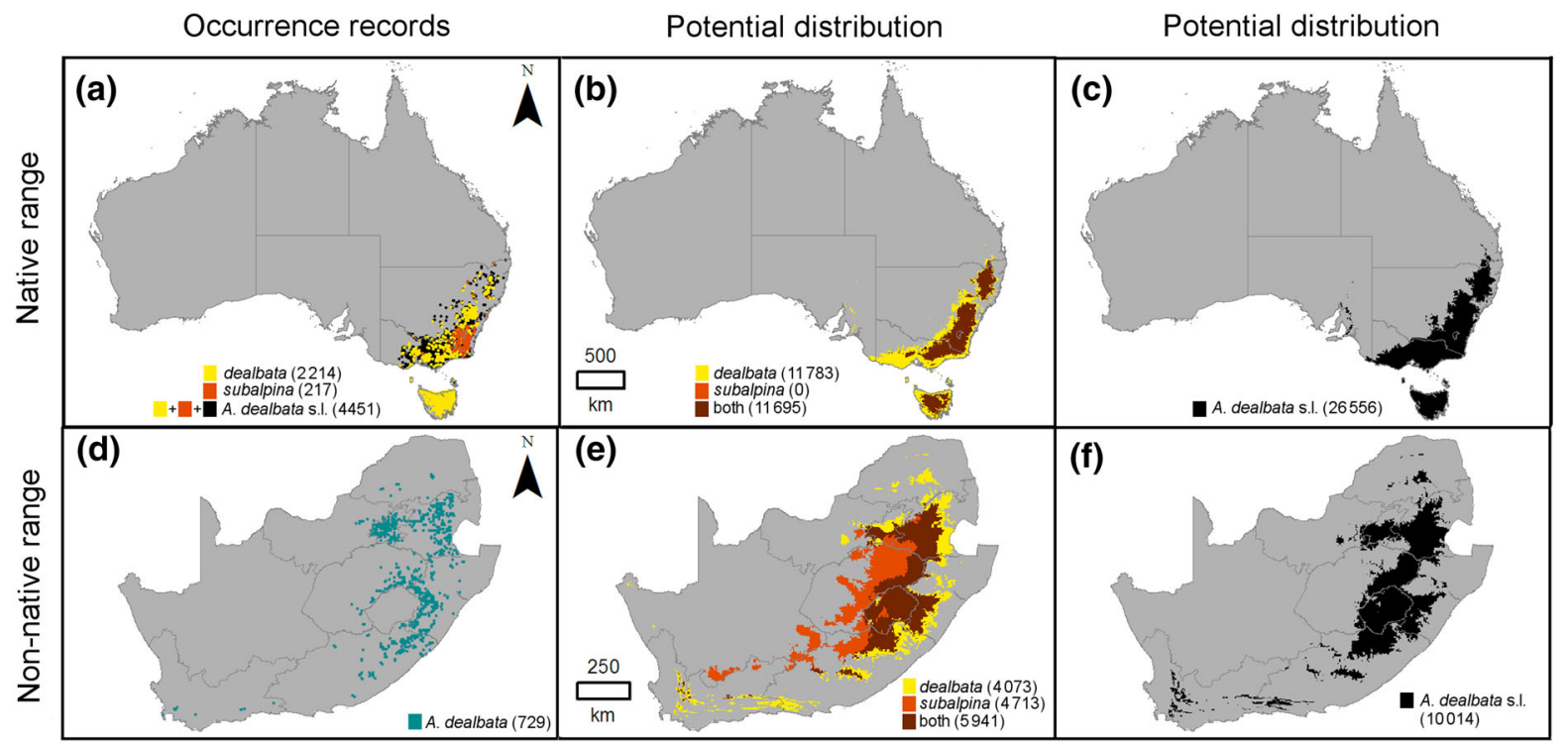

Fig. 1 Binary projections of the ensemble models trained with the different sub-specific datasets of native Acacia dealbata occurrences. The locations of occurrence records used to train the models are represented for the three datasets in panel (a). The second and third panel in the top row show projections in Australia (b, c) and in the bottom row projections in South Africa (incl. Lesotho) (e, f). The projections for the two subspecific models (i.e. ssp. subalpina and ssp. dealbata) are shown together in one map for Australia (b) and one map for South

DNA sequence variation and phylogeographic structure

A total of 18 haplotypes was identified for the 104 samples analyzed (Table S1, Online Resource). Most samples (80.9\%) represented only three haplotypes (I: 22 samples; II: 13 samples; III: 49 samples) which were all shared between both regions of the native range (Australia mainland and Tasmania) as well as the non-native range in South Africa (Fig. 2). Twelve haplotypes were only found in the native range of $A$. dealbata, with two haplotypes unique to Tasmania and ten unique to mainland of Australia (Fig. 2; Table S1, Online Resource). In the non-native range, three haplotypes were identified which were not represented in the native range (haplotypes XVI, XVII, and XVIII; Fig. 2; Table S1, Online Resource). When considering the putative subspecies classification (Table S1, Online Resource), the haplotype network revealed no clear genetic differentiation between the two putative subspecies groups (Fig. 2). In fact, in many
Africa (e). Overlapping areas between the two subspecies (i.e. areas which were projected as suitable by both sub-specific models) are highlighted with a different colour (see colour legends within the panels). The projections of the A. dealbata s.1. ensemble model in Australia and South Africa are shown in panel (c) and (f), respectively. Numbers in parentheses indicate either the sum of grid cells (resolution: 2.5 arc minutes) which were predicted as climatically suitable $(\mathbf{b}, \mathbf{c}, \mathbf{e}, \mathbf{f})$ or the sum of the corresponding distribution records $(\mathbf{a}, \mathbf{d})$

instances the two putative subspecies shared the same haplotypes.

The rarefaction approach revealed a higher haplotype richness $(\mathrm{Hk}=0.739)$ and a remarkable lower absolute gene differentiation (DGst $=0.025)$ in the non-native South African range compared to the both regions within the native range (Australia mainland: $\mathrm{Hk}=0.666$, DGst $=0.075$; Tasmania: $\mathrm{Hk}=0.720$, DGst $=0.080$ ).

\section{Discussion}

Our climatic niche modelling as well as phylogeographic results do not support a taxonomic subdivision of A. dealbata into two distinct subspecies as described by Kodela and Tindale (2001). These results confirm what we observed during field surveys and collections in the native range, where it was not possible to reliably separate the two subspecies based on their physical characteristics as detailed in the 


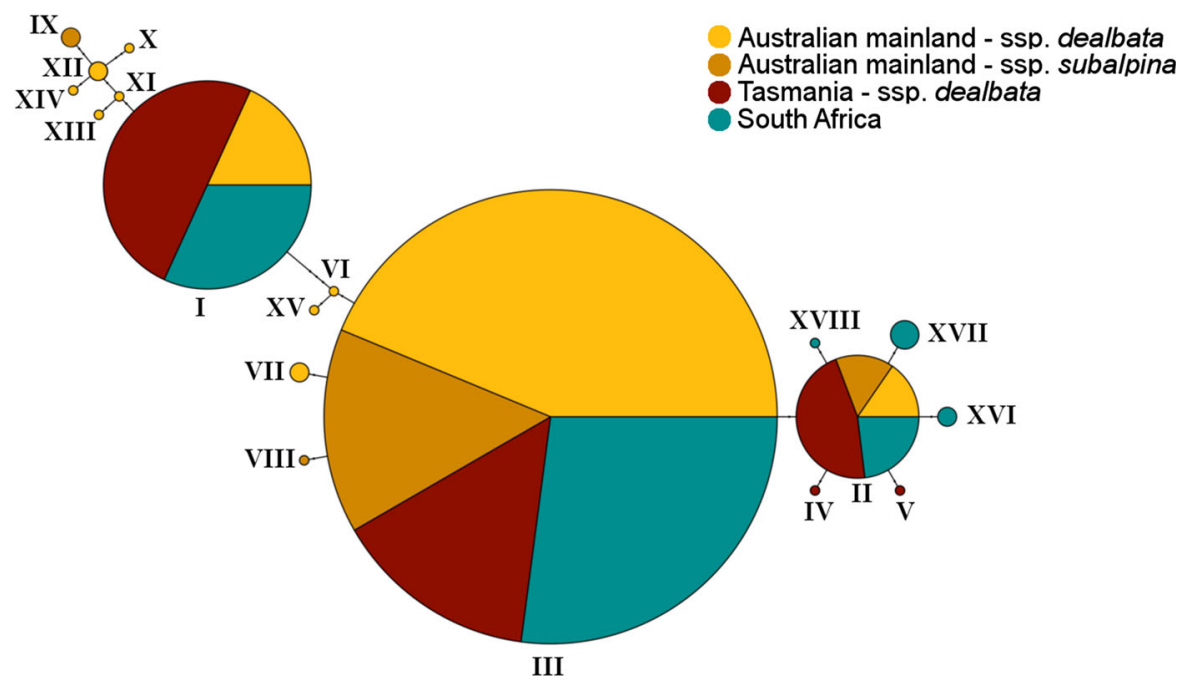

Fig. 2 Parsimony network based on external transcribed spacer (ETS) haplotypes of Acacia dealbata. Colours represent the different geographical regions from where the samples were collected (native range: Australian mainland and Tasmania; non-native range: South Africa) and as well as the subspecies

description by Kodela and Tindale (2001). Indeed, the observations made at higher altitudes, where ssp. subalpina is purported to occur, including the area where the holotype was collected, could not preclude the possibility that any differences being observed were simply due to phenotypic plasticity. Although delayed reproductive phenology occurred at these higher altitudes, it is likely a response to cooler temperatures.

Although the total areas of suitable climatic conditions within the native range differed to some degree between the three ensemble SDMs, a pronounced overlap of those areas makes a clear sub-specific niche differentiation unlikely (even in Tasmania where only ssp. dealbata is reported to occur; Fig. 1b, c). Our assumption is further supported by the observed high predictive power of each ensemble model. We suspect that the lower predictive power of the spp. subalpina model for ssp. dealbata and A. dealbata occurrences is because of the comparatively small number of occurrence records which were available for the calibration of this model rather than mirroring niche differentiations.

We are aware that niche modelling approaches alone may not be efficient to differentiate the two putative subspecies, especially when considering the varying number of occurrence records available for the specific entities. However, Thompson et al. (2011) categories initially assigned (see Table S1 for further details). The visual subspecies differentiation in the native range was only done for the Australian mainland range since it is generally assumed that only ssp. dealbata occurs in Tasmania

found that SDM approaches are indeed able to detect bioclimatic niche differences below the species level for the Acacia saligna species complex. Irrespective of these issues, subspecies would possibly be distinguished by our molecular data as distinct genetic lineages (network clusters). The DNA region we employed here has been regularly used to distinguish between subspecies of various other Australian acacias (e.g. Le Roux et al. 2011; Ndlovu et al. 2013; Thompson et al. 2015). However, in agreement with the bioclimatic models, our DNA sequencing results were unable to identify any distinct lineages, with some haplotypes shared between the two putative subspecies. Even accessions from the highest altitudinal regions sampled $(>1300 \mathrm{~m}$ a.s.l.), where the occurrence of morphological intermediate stands can definitely be discounted, shared haplotypes with samples collected at very low altitudes $(<300 \mathrm{~m}$ a.s.l.; Table S1).

The identification of three high-frequency haplotypes that were shared between samples from across the native range and non-native samples, a high number of unique haplotypes, as well as the higher haplotype richness found in South Africa, indicates that repeated introductions most likely occurred. This assumption is in line with historical information on $A$. dealbata introductions to South Africa, indicating that different native seed lots from Australia were used in 
several forestry trials, and that seeds were secondarily imported from Italy (Poynton 2009). However, a finer scale genetic approach (e.g. single-nucleotide polymorphism or microsatellites) on A. dealbata is needed to determine potential sources of introduction more precisely and to understand the genetic composition of native and non-native populations in more detail.

Moreover, the moderate power of all SDMs to correctly predict occurrences in the non-native range indicates the possibility of a climatic niche shift in the South African range of A. dealbata. For example, Gallien et al. (2016) recently showed evidence of such potential niche shifts during colonization under new climatic conditions using Ambrosia artemisiifolia as model organism. Further research on the potential niche shift in non-native South African A. dealbata populations is necessary to understand the extent of such a shift. Such knowledge is crucial to support ongoing attempts to improve the efficiency of biological control of the species in South Africa. Other forms of control (such as the integrated use of mechanical and chemical methods, fire, and exploitation) have not reduced the extent of invasive populations nor slowed the rate of spread of the species in the country (van Wilgen et al. 2011). This is because of the species' capability to produce vast stores of long-lived seeds that accumulate in the soil and are stimulated to germinate by fire, as well as its ability to resprout vigorously after cutting (Richardson and Kluge 2008; Poynton 2009).

Overall, our study demonstrates that reliable taxonomic classification is important when investigating the invasion ecology of individual species. Our data does not support the notion that $A$. dealbata consists of two distinct subspecies, and therefore inferences on the species' invasion potential, as well as potential native source regions for biological control exploration, might be grossly misled using traditional classification. Our results suggest that invasive populations in South Africa have arisen from multiple introductions and that future biological control attempts should assume that there is no evidence for a sub-specific taxonomic classification of A. dealbata and that biological control agents should be sourced from across the native range of the species.

Acknowledgements Funding was provided by the DST-NRF Centre of Excellence for Invasion Biology and the Working for Water Programme through their collaborative research project on "Integrated Management of invasive alien species in South Africa" and a Subcommittee B grant from Stellenbosch University (to JLR), and the Drakenstein Trust. Additional financial support was provided by the DST-NRF Centre of Excellence for Invasion Biology, Stellenbosch University, and the National Research Foundation of South Africa (Grant Nos. 85417 to DMR). We thank C. Gairifo, J. Ndlovu, and J.R.U. Wilson for collecting and/or providing samples analyzed in this study, and H. Kaplan for supplying occurrence records listed in the Southern African Plant Invaders Atlas. We are grateful to M.J. Koordom and P.H. Du Preez for assistance in the laboratory. We also thank the two anonymous referees whose suggestions helped to clarify and improve this manuscript significantly.

\section{References}

Allouche O, Tsoar A, Kadmon R (2006) Assessing the accuracy of species distribution models: prevalence, kappa and the true skill statistic (TSS). J Appl Ecol 43:1223-1232

Araújo MB, New M (2007) Ensemble forecasting of species distributions. Trends Ecol Evol 22:42-47

Castro-Díez P, Godoy O, Saldana A, Richardson DM (2011) Predicting invasiveness of Australian acacias on the basis of their native climatic affinities, life history traits and human use. Divers Distrib 17:934-945

Early R, Bradley BA, Dukes JS, Lawler JJ, Olden JD, Blumenthal DM, Gonzalez P, Grosholz ED, Ibañez I, Miller LP, Sorte CJB, Tatem AJ (2016) Global threats from invasive alien species in the twenty-first century and national response capacities. Nat Commun 7:12485

Ensing DJ, Moffat CE, Pither J (2013) Taxonomic identification errors generate misleading ecological niche model predictions of an invasive hawkweed. Botany 91:137-147

Gallien L, Thuiller W, Fort N, Boleda M, Alberto FJ, Rioux D, Lainé J, Savergne S (2016) Is there any evidence for rapid, genetically-based, climatic niche expansion in the invasive common ragweed? PLoS ONE 11:e0152867

Guisan A, Zimmermann NE (2000) Predictive habitat distribution models in ecology. Ecol Model 135:147-186

Henderson L (2007) Invasive, naturalized and casual alien plants in southern Africa: a summary based on the Southern African Plant Invaders Atlas (SAPIA). Bothalia 37: 215-248

Hijmans RJ, Cameron SE, Parra JL, Jones PG, Jarvis A (2005) Very high resolution interpolated climate surface for global land areas. Int J Climatol 25:1965-1978

Holmes PM, Richardson DM, Esler KJ, Witkowski ETF, Fourie $S$ (2005) A decision-making framework for restoring riparian zones degraded by invasive alien plants in South Africa. S Afr J Sci 101:553-564

Impson FAC, Kleinjan CA, Hoffmann JH, Post JM, Wood AR, Moran VC (2011) Biological control of Australian Acacia species and Paraserianthes lophantha (Willd.) Nielsen (Mimosaceae) in South Africa. Afr Entomol 19:186-207

Kodela PG, Tindale MD (2001) Acacia dealbata subsp. subalpina (Fabaceae: Mimosiodeae), a new species from south-eastern Australia. Telopea 9:319-322 
Le Roux JJ, Brown GK, Byrne M, Ndlovu J, Richardson DM, Thompson GD, Wilson JRU (2011) Phylogeographic consequences of different introduction histories of invasive Australian Acacia species and Paraserianthes lophantha (Fabaceae) in South Africa. Divers Distrib 17:861-871

Marmion M, Parviainen M, Luoto M, Heikkinen RK, Thuiller W (2009) Evaluation of consensus methods in predictive species distribution modelling. Divers Distrib 15:59-69

Maslin BR, McDonald MW (2004) Acacia search. Evaluation of Acacia as a woody crop option for southern Australia. Rural Industries Research and Development Corporation, Canberra

Ndlovu J, Richardson DM, Wilson JRU, O’Leary M, Le Roux JJ (2013) Elucidating the native sources of an invasive tree species, Acacia pycnantha, reveals unexpected native range diversity and structure. Ann Bot 111:895-904

Paradis E (2010) pegas: an R package for population genetics with an integrated-modular approach. Bioinformatics 26:419-420

Paterson ID, Hoffmann JH, Klein H, Mathenge CW, Neser S, Zimmermann HG (2011) Biological control of Cactaceae in South Africa. Afr Entomol 19:230-246

Petit RJ, El Mousadik A, Pons O (1998) Identifying populations for conservation on the basis of genetic markers. Conserv Biol 12:844-855

Poynton RJ (2009) Tree planting in southern Africa. Other genera, vol 3. Department of Agriculture, Forestry and Fisheries, Pretoria, South Africa

Pyšek P, Hulme PE, Meyerson LA, Smith GF, Boatwright JS, Crouch NR, Figueiredo E, Foxcroft LC, Jarošik V, Richardson DM, Suda J, Wilson JRU (2013) Hitting the right target: taxonomic challenges for, and of, plant invasions. AoB PLANTS 5:plt042

R Core Team (2016) R: A language and environment for statistical computing. R Foundation for Statistical Computing, Vienna, Austria. https://www.R-project.org/

Rejmánek M, Richardson DM (2013) Trees and shrubs as invasive alien species-2013 update of the global database. Divers Distrib 19:1093-1094
Richardson DM, Kluge RL (2008) Seed banks of invasive Australian Acacia species in South Africa: role in invasiveness and options for management. Perspect Plant Ecol Syst 10:161-177

Robertson MP, Visser V, Hui C (2016) Biogeo: an R package for assessing and improving data quality of occurrence record datasets. Ecography 39:394-401

Saltonstall K (2002) Cryptic invasion by a non-native genotype of the common reed, Phragmites australis, into North America. Proc Natl Acad Sci USA 99:2445-2449

Swets KA (1988) Measuring the accuracy of diagnostic systems. Science 240:1285-1293

Thompson GD, Robertson MP, Webber BL, Richardson DM, Le Roux JJ, Wilson JRU (2011) Predicting the subspecific identity of invasive species using distribution models: Acacia saligna as an example. Divers Distrib 17:10011014

Thompson GD, Bellstedt DU, Richardson DM, Wilson JRU, Le Roux JJ (2015) A tree well travelled: global genetic structure of the invasive tree Acacia saligna. J Biogeogr 42:305-314

Thuiller W, Georges D, Engler R, Breiner F (2016) biomod2: Ensemble platform for species distribution modeling. $\mathrm{R}$ package version 3.3-7. https://CRAN.R-project.org/pack age=biomod 2

Van Wilgen BW, Dyer C, Hoffmann JH, Ivey P, Le Maitre DC, Richardson DM, Rouget M, Wannenburgh A, Wilson JRU (2011) National-scale strategic approaches for managing introduced plants: insights from Australian acacias in South Africa. Divers Distrib 17:1060-1075

Wardill TJ, Graham GC, Zalucki M, Palmer WA, Playford J, Scott KD (2005) The importance of species identity in the biocontrol process: identifying the subspecies of Acacia nilotica (Leguminosae: Mimosoideae) by genetic distance and the implications for biological control. J Biogeogr 32:2145-2159 